\title{
A DOMESTICATED CODE: ON THE EMERGENCE OF THE OSCILLATORY BASIS OF PHRASE STRUCTURE
}

\author{
ELLIOT MURPHY*1,2 \\ *Corresponding Author: elliot.murphy.13@ucl.ac.uk \\ ${ }^{1}$ Division of Psychology and Language Sciences, University College London, England \\ ${ }^{2}$ Department of Psychology, University of Westminster, England
}

The search for the 'neural code' in animal neurophysiology has recently seen a marked transition from the analysis of individual spike timings to larger patterns of synchronisation, and it will be argued that the study of language should readily embrace these systems-level developments in the field. An emerging consensus in linguistics is that the language system needs to exploit two memory workspaces in order to generate and maintain/interpret non-local syntactic relations (e.g. Adger, 2017). One workspace is used to construct syntactic phrases, and the other is used to maintain these units. The findings of a number of MEG studies will be discussed in order to suggest that an initial phase-amplitude coupling $\theta-\gamma$ code constitutes the first workspace, and a subsequently embedded $\delta-\theta$ code constitutes the second. It will be shown that this account goes some way to explain why leftinferior frontal regions seem sensitive to syntactic violations, and why anterior temporal regions seem to be implicated in semantic composition operations.

If the particular $\delta-\theta$ interactions documented here are indeed humanspecific, this could derive the apparent uniqueness of the dual workspace of human syntax. $\delta$ appears vital for language comprehension, but from a neurocomputational perspective, cross-frequency coupling involving $\delta$ may also play a vital role in the online construction of phrases, instead of 'passively' entraining to phrasal units (Ding et al., 2016). Although cross-frequency coupling is most commonly found between $\theta$ and $\gamma$, recent work suggests that $\theta$ entrains to $\delta$ in the human brain in a uniquely diverse way (Maris et al., 2016). Given experimental findings that these slow rhythms entrain to sentential and phrasal structures (Mai et al., 2016; Bradley \& Iverson, 2017), the discovery of humanspecific forms of $\delta-\theta$ phase-amplitude coupling is potentially of great significance; in particular when interpreted alongside findings that non-human primate call systems exhibit limited cross-frequency couplings, as shown in Murphy (2016a-c, Forthcoming a,b). Grimaldi (2017: 17) summarises that 'Murphy [(2016b)] proposes a revolutionary theory of neurolinguistics ... that 
nested oscillations execute elementary linguistic computations', adding that this theory 'goes considerably beyond existing models'. This model will be refined, taking into account recent findings concerning, for instance, travelling oscillations.

It will further be argued that this phase code can be explained through reference to the domestication syndrome and the 'globularity' hypothesis (Benítez-Burraco et al., 2017), such that the human braincase permits a reduction in 'spatial inequalities' (Salami et al., 2003) between cortical and subcortical regions documented in less globular brains. The MEG data presented will be shown to be in line with this position.

In addition, a critique of recent neuroethological work is presented. For instance, Kikuchi et al. (2017) claim to find no differences in the cross-frequency coupling profiles of humans and monkeys, however they only examine coupling between low frequencies and $\gamma$, and not between low frequencies such as $\delta, \theta$ and $\beta$. Upon its emergence, the human-specific oscillatory profile would have interfaced with more ancient systems shared with other primates, in turn influencing and modifying the internal organisation of such systems through a process of 'reciprocal causation' (Walsh 2015). As a result, the emergence of the presently proposed neural code would likely have re-shaped the representations of the conceptual systems it interfaced with. It is argued that this can explain why primate call units appear to be lacking in the diverse array of language-specific representations. More specifically, it is argued that domestication can be used as a model system for the Extended Evolutionary Synthesis (Zeder, 2017) and can enhance our understanding of reciprocal causation and niche-construction, which in turn allows neurolinguistics to make more direct contact with the life sciences.

Lastly, the potential for cross-frequency coupling to derive the computational complexity of hierarchically organised phrase structures is explored in order to construct a set of working hypotheses concerning the role of particular neural oscillations in linguistic computation. Processes once deemed highly domain specific - from grid cells to the theta-gamma code for working memory - are increasingly being shown to execute generic computations operating over items stored across distinct representational domains (Constantinescu et al., 2016; Kriegeskorte \& Storrs, 2016; Lisman \& Jensen, 2013), and the consequences of this for the language sciences are presented. It will be argued that a number of recent studies claiming to make substantial insights into the oscillatory nature of language have engaged primarily in data redescription rather than theory-construction, and this discussion will be used to motivate a neurobiologically feasible model of syntactic computation which brings with it direct consequences for more traditional, cartographic models.

\section{Acknowledgements}


This work was supported by an Economic and Social Research Council scholarship (1474910).

\section{References}

Adger, D. (2017). A memory architecture for merge. Ms. Queen Mary University. Benítez-Burraco, A., Di Pietro, L., Barba, M., \& Lattanzi, W. (2017). Schizophrenia and human self-domestication: an evolutionary linguistics approach. Brain, Behavior and Evolution, 89(3), 162-184.

Bradley, H., \& Iverson, P. (2017). How do infants process speech in the mind? An EEG study of neural entrainment and the Frequency Following Response. Poster presented at the UCL MRes/MSc Language Sciences Poster Session.

Constantinescu, A. O., O'Reilly, J. X., \& Behrens, T. E. J. (2016). Organizing conceptual knowledge in humans with a gridlike code. Science, 352(6292), 1464-1468.

Ding, N., Melloni, L., Zhang, H., Tian, X., \& Poeppel, D. (2016). Cortical tracking of hierarchical linguistic structures in connected speech. Nature Neuroscience, 19, 158-164.

Kikuchi, Y., Attaheri, A., Wilson, B., Rhone, A. E., Nourski, K. V., Gander, P. E., Kovach, C. K., Karasaki, H., Griffiths, T. D., Howard III, M. A., \& Petkov, C. I. (2017). Sequence learning modulates neural responses and oscillatory coupling in human and monkey auditory cortex. PLOS Biology, 15(4), e2000219.

Kriegeskorte, N., \& Storrs, K. R. (2016). Grid cells for conceptual space? Neuron, 92, 280-284.

Lisman, J. E., \& Jensen, O. (2013). The theta-gamma neural code. Neuron, 77, 1002-1016.

Mai, G., Minett, J. W., Wang, W. S.-Y (2016) Delta, theta, beta, and gamma brain oscillations index levels of auditory sentence processing. NeuroImage, 113, 516-528.

Maris, E., Fries, P., \& van Ede, F. (2016). Diverse phase relations among neuronal rhythms and their potential function. Trends in Neurosciences, 39(2), 86-99.

Murphy, E. 2016a. Evolutionary monkey oscillomics: Generating linking hypotheses from preserved brain rhythms. Theoretical Linguistics, 42(1-2), 117-137.

Murphy, E. 2016b. The human oscillome and its explanatory potential. Biolinguistics, 10, 6-20.

Murphy, E. 2016c. A theta-gamma neural code for feature set composition with phase-entrained delta nestings. UCL Working Papers in Linguistics, 28, 1-23.

Murphy, E. Forthcoming a. Principles of Language Evolution. [Textbook]. London: UCL Press.

Murphy, E. Forthcoming b. Implications of travelling weakly coupled oscillators for the cortical language circuit. UCL Working Papers in Linguistics. 
Salami, M., Itami, C., Tsumoto, T., Kimura, F. (2003). Change of conduction velocity by regional myelination yields constant latency irrespective of distance between thalamus and cortex. PNAS, 100, 6174-6179.

Walsh, D. M. (2015). Organisms, Agency, and Evolution. Cambridge: Cambridge University Press.

Zeder, M. A. (2017). Domestication as a model system for the extended evolutionary synthesis. Interface Focus, 7, 20160133. 